\title{
Assessment of nephrotoxicity of herbal medicine containing aristolochic acid in mice
}

\author{
Yi Quan ${ }^{1,2,}$, Long Jin ${ }^{1,2,}$, Kang Luo ${ }^{1,2}$, Jian Jin ${ }^{1,2}$, Sun Woo Lim ${ }^{1,2}$, Yoo Jin Shin ${ }^{1,2}$, Eun Jeong Ko ${ }^{2,3}$, \\ Byung Ha Chung ${ }^{2,3}$, and Chul Woo Yang ${ }^{1,3}$
}

${ }^{1}$ Transplant Research Center, ${ }^{2}$ Convergent Research Consortium for Immunologic Disease, ${ }^{3}$ Division of Nephrology, Department of Internal Medicine, Seoul St. Mary's Hospital, College of Medicine, The Catholic University of Korea, Seoul, Korea
Received: July 30, 2018

Revised : October 4, 2018

Accepted: November 19, 2018

\section{Correspondence to}

Chul Woo Yang, M.D.

Division of Nephrology,

Department of Internal

Medicine, College of Medicine,

Seoul St. Mary's Hospital, The

Catholic University of Korea, 222

Banpo-Daero, Seocho-gu, Seoul,

06591, Korea

Tel: +82-2-2258-6851

Fax: +82-2-2258-6917

E-mail:yangch@catholic.ac.kr

*'These authors contributed equally to this work.
Background/Aims: It is undetermined if herbal medicines (HM) containing aristolochic acid (AA)-containing have similar nephrotoxicity to AA itself.

Methods: We administered HM containing a high concentration of AA for 5 days (short-term study) or a low concentration of AA for 30 days (long-term study) to $\mathrm{C}_{57} \mathrm{BL} / 6$ mice; for comparison, same dose of AA compound was used as controls.

Results: The nephrotoxicity in the HM- and AA-treated mice was compared in terms of renal function, histopathology, oxidative stress, apoptotic cell death, and mitochondrial damage. Short-term HM treatment resulted in acute kidney injury (marked renal dysfunction, acute tubular necrosis, and neutrophil gelatinase-associated lipocalin [NGAL] expression) in which the severity of renal dysfunction and histopathology was comparable with that induced by the administration of AA alone. Long-term HM treatment resulted in features of chronic kidney disease (CKD, mild renal dysfunction and tubular atrophy and dilatation). No significant differences in these parameters were observed between the HM- and AA-treated mice. HM-induced oxidative stress (8-hydroxy-2'-deoxyguanosine and manganese-dependent superoxide dismutase expression) and apoptotic cell death (terminal deoxynucleotidyl transferase dUTP nick end labelling [TUNEL]-positive cells and active caspase-3 expression) were similar in HM- and AA-treated mice in the short-term and long-term studies. Mitochondrial injury, evaluated by electron microscopy, was also similar in HM- and AA-treated mice in the short-term and long-term studies.

Conclusions: The nephrotoxic potential of HM containing AA was similar to that of AA itself.

Keywords: Herbal medicine; Aristolochic acid; Oxidative stress; Apoptosis

\section{INTRODUCTION}

Aristolochic acid (AA)-containing compounds gained notoriety after the emergence of interstitial nephritis in European women who took these compounds to achieve weight loss [1]. The first case of aristolochic acid nephropathy (AAN) was reported in Belgium, and several cases of irreversible renal toxicity after intake of AA-containing compounds have since been reported [1-3].

The presence of AA in an herbal medicine (HM) is important for the diagnosis of AAN in patients with HM-induced renal dysfunction [4]. However, it has not been confirmed that the nephrotoxic potential of AA-containing HM is similar to the nephrotoxic poten- 
tial of AA. Furthermore, it is unknown if the presence of $\mathrm{AA}$ in $\mathrm{HM}$ is a major causative factor of renal injury. Therefore, the goal of our study was to evaluate the nephrotoxic potential of HM containing AA in an experimental model in mice [5].

\section{METHODS}

\section{Characteristics of HM used in experiment}

We obtained HM from a 33-year-old woman who was prescribed HM for postpartum edema control for one and half months at oriental private clinic. Five months later, routine health examination showed normal renal function and mild proteinuria. Four months later, she visited emergency room due to flank pain, fever and poor oral intake. Laboratory findings revealed renal failure (blood urea nitrogen $68.4 \mathrm{mg} / \mathrm{dL}$, serum creatinine $8.98 \mathrm{mg} / \mathrm{dL}$ ), anemia (hemoglobin $8.0 \mathrm{~g} / \mathrm{dL}$ ) and metabolic acidosis ( $\mathrm{pH} 7.306$, bicarbonate $15.4 \mathrm{mEq} / \mathrm{L}$ on arterial blood gas analysis). We started hemodialysis due to uremic symptoms and performed renal biopsy. Pathologic finding was chronic tubulointerstitial nephritis. Thus, we suspected Chinese herb nephropathy, and confirmed AAN by the presence of AA $(21.75 \mathrm{mg} / \mathrm{kg})$ in HM using high-performance liquid chromatography.

\section{Experimental animals and AAN model design}

The Animal Care and Use Committee of the Catholic University of Korea approved the experimental protocol (CUMS-2017-0218-01). All procedures performed in this study followed the appropriate ethical guidelines for animal studies. Six-week-old $\mathrm{C}_{57} \mathrm{BL} / 6$ male mice (Orient Bio, Seongnam, Korea), weighing 21 to $23 \mathrm{~g}$, were housed in individual cages at a constant temperature and under a controlled light cycle (12 hours light/12 hours dark). The mice were fed a normal diet with sterilized tap water.

After all mice were acclimatized for 1 week, weightmatched mice were randomized to six groups $(n=8 /$ group): three groups were used for the short-term study and three groups were used for the long-term study. In the short-term study, HM was made to final concentrations of AA $(5 \mathrm{mg} / \mathrm{kg})$ and intraperitoneally injected to the mice once per day for 5 days. For the long-term study, we calculated the patient's daily intake of AA ( $0.073 \mathrm{mg} / \mathrm{kg}$ twice a day) in $\mathrm{HM}(200 \mathrm{~mL} /$ day/60 kg, twice a day; AA content, $4.35 \mathrm{mg} / 200 \mathrm{~mL}$ ), and treated HM or AA via oral gavage, twice per day for 30 days. AA (Sigma-Aldrich, St. Louis, MO, USA) was diluted in phosphate-buffered saline (PBS) and sterilized tap water was given to the $\mathrm{VH}$ group. After the short-term or long-term treatment, the animals were anesthetized, and blood samples and tissue specimens were obtained for further analysis.

\section{Measurement of renal function}

Blood urea nitrogen (BUN) and serum creatinine ( $\mathrm{SCr}$ ) were measured by using a quantitative enzyme colorimetric method (Stanbio Laboratory, Boerne, TX, USA) in accordance with the manufacturer's instructions.

\section{Evaluation of renal histology}

For conventional microscopy of the animals in the short-term study, fixed kidney samples embedded in wax were cut into $5 \mu \mathrm{m}$ slices and stained with hematoxylin and eosin reagents. A complete section of the kidney was screened at a magnification of $\times 400$, and findings for the cortex were semi-quantitatively scored by three independent observers. We assessed renal tubular necrosis by measuring the randomly selected hematoxylin and eosin (H\&E) stained sections, and presented its severity as scores based on the percentage of tubules affected (o: < 10\%; 1: $10 \%$ to $25 \%$; 2 : $25 \%$ to $50 \% ; 3: 50 \%$ to $75 \%$; $4:>75 \%$ ) [6]. For the analysis of the mice in the long-term study, kidney tissue sections were stained with periodic acid-Schiff reagent. The tubular dilatation was estimated in a minimum of 20 fields per section by counting the percentage of dilated area per field at a magnification of $\times 400$ using the polygon program (TDI Scope Eye version 3.6 for windows, Olympus, Seoul, Korea). Histopathologic analysis was performed in randomly selected fields of sections by a pathologist blinded to the identity of the treatment groups.

\section{Immunohistochemistry analyses and TUNEL staining} Immunohistochemistry was performed to identify markers of tubular injury (neutrophil gelatinase-associated lipocalin [NGAL]), oxidative stress (8-hydroxy-2'-deoxyguanosine [8-OHDG]) and apoptosis (active caspase 3) using previously described methods [7]. 
Primary antibodies for immunohistochemistry are as follows: NGAL antibody (Millipore, Bellerica, MA, USA) 8-OHDG (JaICA, Shizuoka, Japan), manganese-dependent superoxide dismutase (MnSOD, Abcam, Cambridge, MA, USA) and active caspase 3 (Millipore, Bellerica, MA, USA). We counted the positive cells per field in 15 randomly selected areas per animal in all groups.

Dewaxed sections were incubated in retrieval solution ( $\mathrm{pH}$ 6.0), methanolic $\mathrm{H}_{2} \mathrm{O}_{2}$, and $0.5 \%$ Triton X-100, and then washed in PBS. Nonspecific binding sites were blocked by incubation in 10\% normal donkey serum (Jackson ImmunoResearch, West Grove, PA, USA). The sections were incubated overnight at $4^{\circ} \mathrm{C}$ with primary antibodies and then with peroxidase-conjugated secondary antibodies (Molecular Probes, Carlsbad, CA, USA; Jackson ImmunoResearch) for 2 hours at room temperature. Peroxidase activity was detected by using 3,3'-diaminobenzidine (Vector Laboratories, Burlingame, CA, USA) as a chromogen. Quantitative analysis was achieved through the calculation of the percentage of positively stained area with the same intensity by using histogram equalization (TDI Scope Eye). Apoptotic cells in tissue sections were detected by the terminal deoxynucleotidyl transferase dUTP nick end labelling (TUNEL) method and staining with the in situ Apoptosis Detection Kit (Millipore, Billerica, MA, USA). For each treatment condition, TUNEL-positive cells were counted in 15 randomly selected, non-overlapping areas per animal.

\section{Immunoblotting analysis}

Whole cells were lysed in PRO-PREP protein-extraction solution (Intron Biotechnology, Seongnam, Korea) in accordance with the manufacturer's instructions. Equal amounts of protein were subjected to immunoblotting analysis; after incubation with the appropriate primary and secondary antibodies, signals were detected by using an enhanced chemiluminescence system (ATTO Corp., Tokyo, Japan). Quantification of the relative band densities was performed; the intensity of the control group was set to $100 \%$ and band densities were normalized to the density of $\beta$-actin bands from the same gel (Quantity One version 4.4.0, Bio-Rad, Hercules, CA, USA).

\section{Electron microscopy}

Renal cortex tissues were fixed in $2.5 \%$ glutaraldehyde in o.1 $\mathrm{M}$ phosphate buffer, were post-fixed with $1 \% \mathrm{O}_{\mathrm{S}} \mathrm{O}_{4}$ and embedded in Epon 812. Ultrathin sections were cut, stained with uranyl acetate/lead citrate, and photographed by using a JEM-120oEX transmission electron microscope (JEOL Ltd., Tokyo, Japan). Sections were scanned randomly at 20 different spots per sample at $x$ 5,000 magnification. The number of mitochondria was measured in 15 random proximal tubular cells by using imaging software (TDI Scope Eye).

\section{Statistical analysis}

The data are expressed as the mean \pm standard error (SE) of at least three independent experiments. Multiple comparisons between groups were performed by oneway analysis of variance with Bonferroni's post hoc test computed by using SPSS software version 19.0 (IBM Co., Armonk, NY, USA). Results with $p$ values of $<0.05$ were considered statistically significant.

\section{RESULTS}

\section{Renal function of AA- and HM-treated mice}

The renal function of each experimental group is shown in Fig. ${ }_{1 A}$ and $1 B$. In the short-term study, $\mathrm{SCr}$ and BUN were markedly increased in the $\mathrm{AA}(\mathrm{SCr}, 0.31 \pm 0.02 \mathrm{mg}$ ) dL; BUN: $173 \pm 7 \mathrm{mg} / \mathrm{dL}$ ) and HM (SCr, $0.32 \pm 0.02 \mathrm{mg} /$ $\mathrm{dL} ; \mathrm{BUN}, 104 \pm 0.1 \mathrm{mg} / \mathrm{dL}$ ) groups compared with the $\mathrm{VH}$ group (SCr, $0.10 \pm 0.01 \mathrm{mg} / \mathrm{dL}$; BUN, $21 \pm 0.2 \mathrm{mg} / \mathrm{dL}$ ) group. In the long-term study, $\mathrm{SCr}(\mathrm{AA}, 0.28 \pm 0.05 \mathrm{mg} / \mathrm{dL}$ vs. $\mathrm{VH}$, $0.16 \pm 0.06 \mathrm{mg} / \mathrm{dL}, p<0.05)$ and BUN (AA, $36 \pm 3.4 \mathrm{mg} / \mathrm{dL}$ vs. VH, $17 \pm 3 \mathrm{mg} / \mathrm{dL}, p<0.05)$ were significantly higher in the AA group than the VH group. Whereas SCr did not increase in the HM group and only a mild increase of BUN was observed ( $23 \pm 1 \mathrm{mg} / \mathrm{dL}, p<0.05$ vs. the $\mathrm{VH}$ group).

\section{Histopathology of the kidneys of AA- or HM-treated mice}

As shown in Fig. ${ }_{1} \mathrm{C}$ and ${ }_{1} \mathrm{D}$, the short-term study revealed a similar severity of renal tubular necrosis in the HM and AA groups $(2.3 \pm 0.3$ vs. $3.2 \pm 0.3, p>0.05)$. The long-term study also indicated a similar extent of tissue injury in the HM and AA groups $(1.9 \pm 0.2$ vs. $3.3 \pm 0.4, p$ $>0.05$ ) (Fig. 1E). The NGAL expression in HM (14 \pm 2 per field) and AA (15.5 \pm 1 per field) groups were increased compared to the $\mathrm{VH}(0.8 \pm 0.02$ per field $)$ group $(p<0.05)$ 


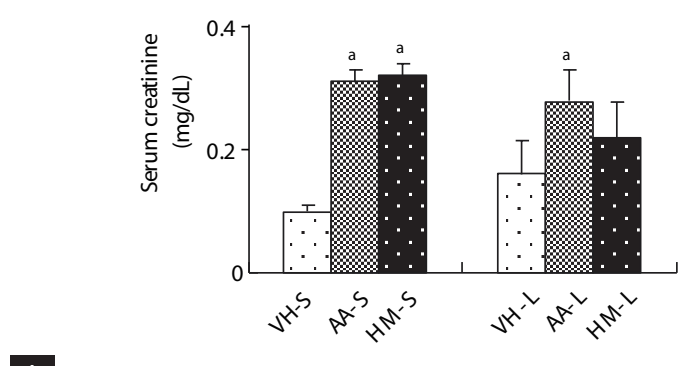

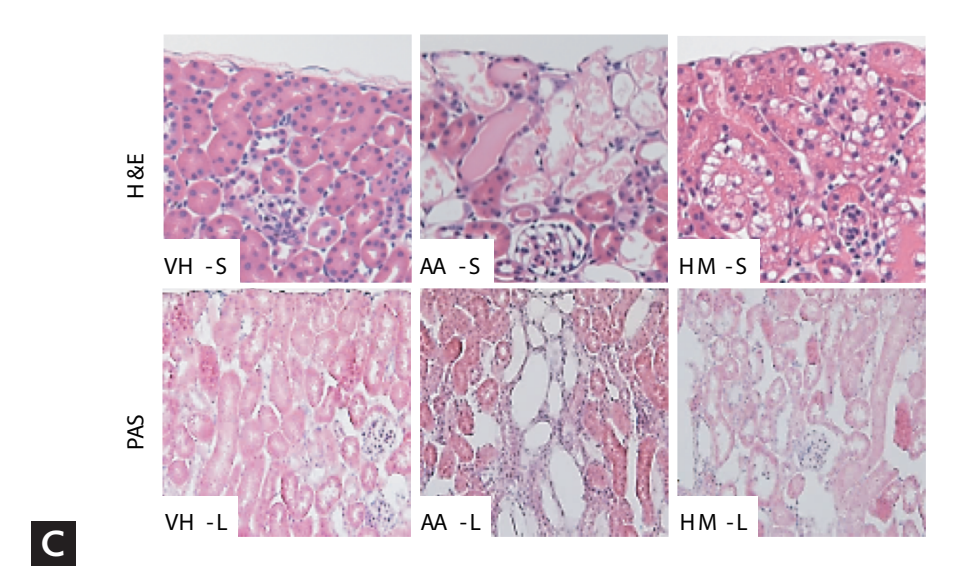

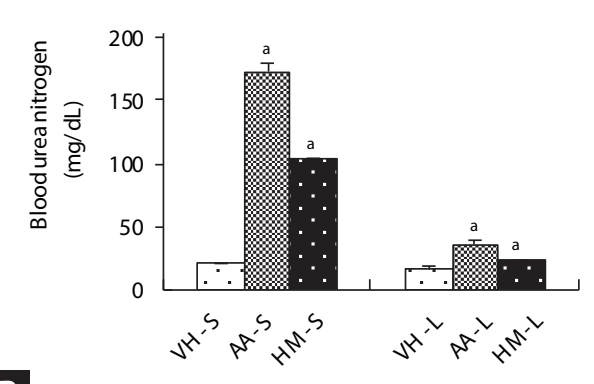

B

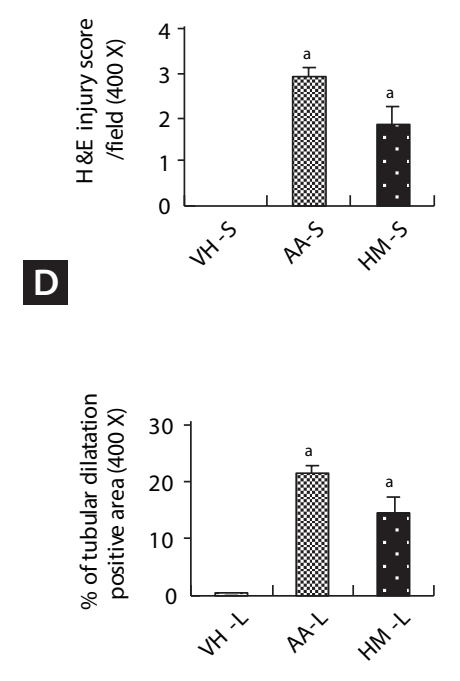

$\mathbf{E}$
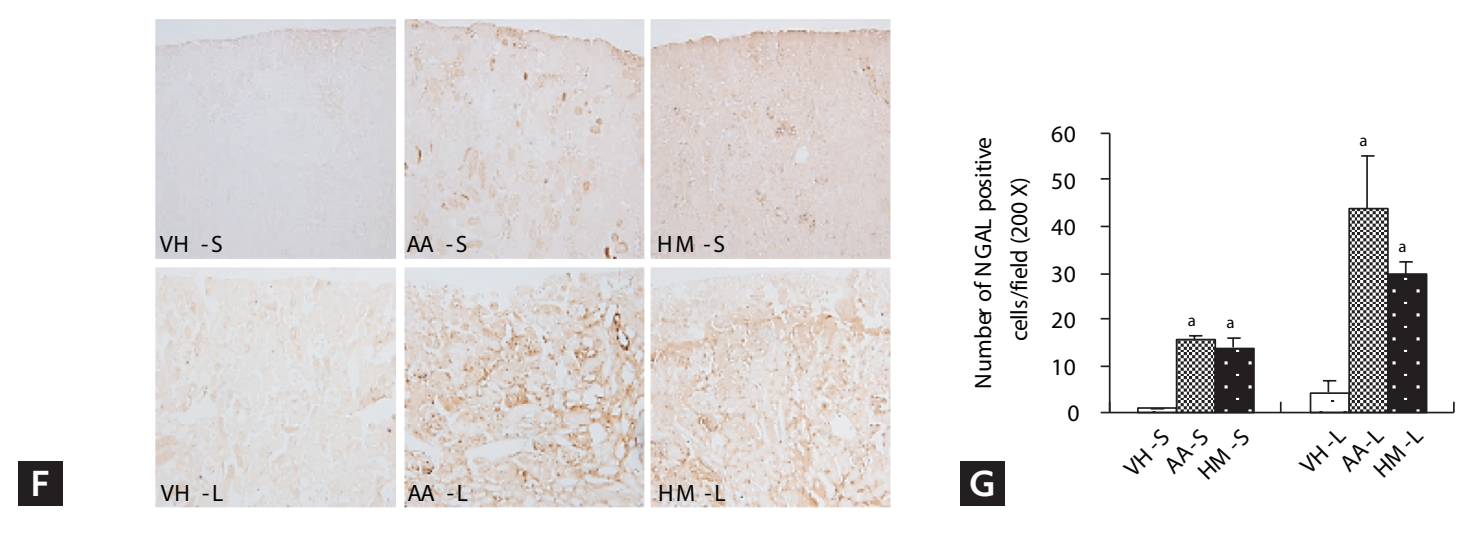

Figure 1. Comparison of renal function and histopathology in aristolochic acid (AA)- and herb medicine (HM)-treated mice in the short-term and long-term studies. (A) Serum creatinine level. (B) Blood urea nitrogen level. (C, D) Representative images of hematoxylin and eosin (H\&E) staining and tissue injury score $(\times 200)$. (C, E) Representative images of periodic acid-Schiff(PAS) stain and quantification graph of tubular dilatation area. (F, G) Representative images of neutrophil gelatinase-associated lipocalin (NGAL) immunohistochemical staining and quantification graph of the NGAL-positive areas. The data are presented as the mean \pm SE. VH, vehicle; $\mathrm{S}$, short-term treatment; L, long-term treatment. ${ }^{\mathrm{a}} \mathrm{A} p<0.05$ vs. the corresponding VH group. 


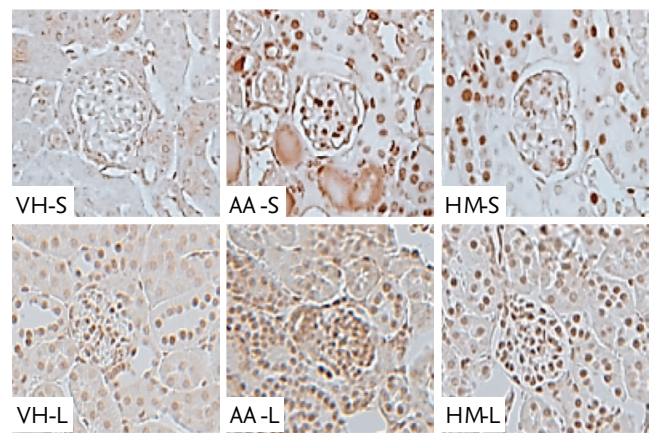

A

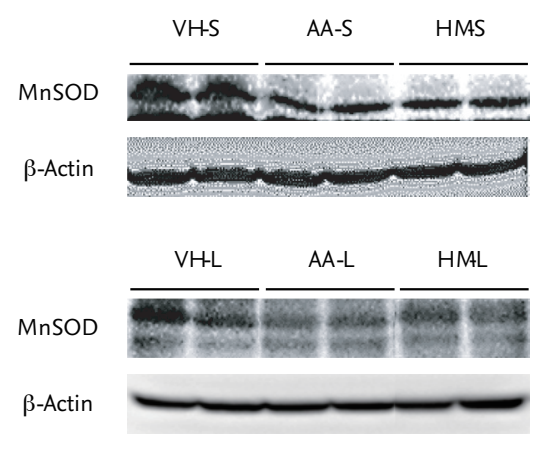

C

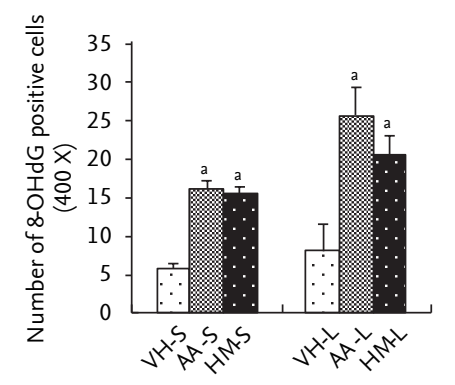

B

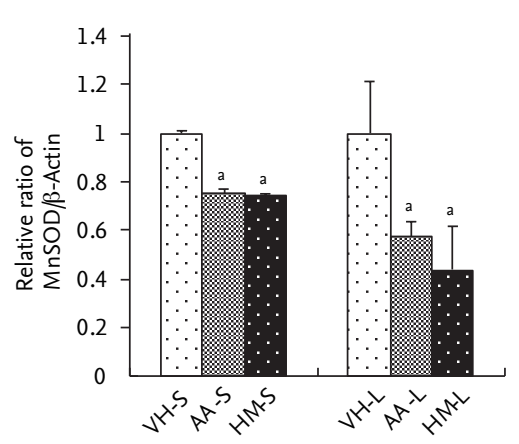

D
Figure 2. Comparison of oxidative stress in aristolochic acid (AA)and herb medicine (HM)-treated mice in the short-term and longterm studies. (A, B) Representative images and numbers of cells positively stained for 8-hydroxy-2'-deoxyguanosine (8-OHdG) in kidney tissue sections. (C, D) Representative immunoblotting and quantification of manganese-dependent superoxide dismutase (MnSOD) in the kidney tissues. The expression was normalized to $\beta$-actin ( $\times 400)$. The data are presented as the mean \pm SE. VH, vehicle; $S$, short-term treatment; L, long-term treatment. ${ }^{\mathrm{a}} \mathrm{A} p<0.05$ vs. the corresponding VH group. in the short-term study. Also in the long-term study, the NGAL expression in HM (29.5 \pm 2.9 per field) and AA (43.8 \pm 11.3 per field) groups were increased compared to the VH $(4.3 \pm 2.5$ per field) group $(p<0.05)$. However, there was no significant difference between the AA and HM groups in short- and long-term studies (Fig. 1 F and $1 \mathrm{G}$ ).

\section{Oxidative stress in the kidneys of AA- or HM-treated mice}

To compare the oxidative stress induced by HM and AA treatments, we measured 8-OHdG and MnSOD in the treatment groups. In the short-term study, the expression of 8-OHdG was significantly higher in the HM (15.6 \pm 0.8 per field) and AA (16.2 \pm 1 per field) groups than in the $\mathrm{VH}(5.8 \pm 0.6$ per field)-treated group $(p<0.05)$, but there was no significant difference between the HM and AA groups. Similar findings were observed in the longterm study (Fig. 2A and 2B).
In short-term study, the relative expression of $\mathrm{Mn}$ SOD was lower in the HM ( $0.74 \pm 0.01$ folds $)$ and AA ( 0.75 \pm 0.02 folds) groups than the VH group $(p<0.05)$, but there was no significant difference between the HM and AA groups. Similar findings were observed in the longterm study (Fig. 2C and 2D).

\section{Mitochondrial injury in the kidneys of AA- or HM-treated mice}

In the AA- and HM-treated groups, proximal tubular cells exhibited severe injury, with altered brush border, cell detachment, and extensive cytoplasmic vacuolization compared with VH treatment (Fig. 3A). The number of mitochondria was lower in both the AA and HM group than the VH group, but there was no significant difference between the AA and HM groups in the short-term $(197 \pm 14$ per field vs. $180 \pm 12$ per field) and long-term (162 \pm 17 per field vs. $180 \pm 10$ per field) studies (Fig. 3B). 

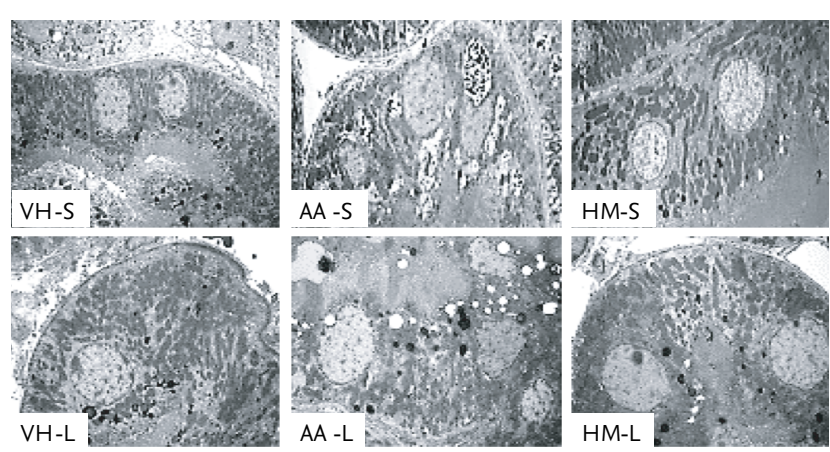

A

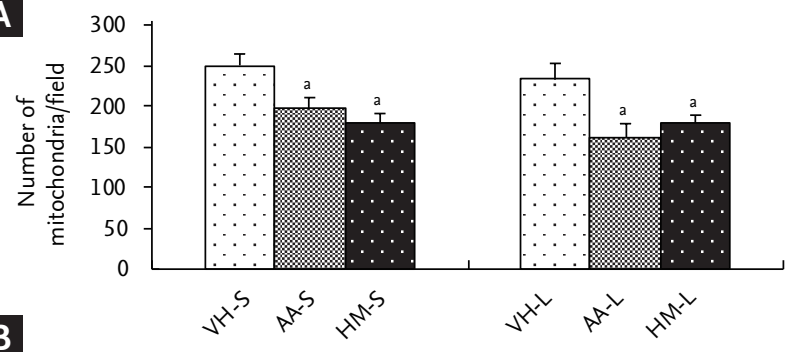

Figure 3. Comparison of mitochondrial injury in the proximal tubules of aristolochic acid (AA) and herb medicine (HM) in the short-term and long-term studies. (A, B) Representative transmission electron microscopy images of the mitochondrial ultrastructure and quantification of the number of mitochondria in the proximal tubules of kidney tissue from mice $\left(x_{5}, 000\right)$. The data are presented as the mean \pm $\mathrm{SE}$. VH, vehicle; S, short-term treatment; L, long-term treatment. ${ }^{a} \mathrm{~A} p<0.05$ vs. the corresponding $\mathrm{VH}$ group.

\section{Apoptosis in the kidney of AA- and HM-treated mice}

The number of TUNEL-positive cells (Fig. $4 \mathrm{~A}$ and $4 \mathrm{~B}$ ) in the $\mathrm{HM}$ and AA groups were markedly higher than the VH group in the short-term $(6.8 \pm 0.4$ per field in the AA group and $6.0 \pm 0.3$ per field in the HM group vs. $1.2 \pm$ 0.8 per field in the $\mathrm{VH}$ group, $p<0.05 \mathrm{vs}$. VH groups) and long-terms studies $(13.6 \pm 3.5$ per field in the AA group and $11.4 \pm 3.8$ per field in the HM group vs. $0.7 \pm 1.2$ per field in the VH group, $p<0.05$ vs. VH groups). However, there was no significant difference between the AA and $\mathrm{HM}$ groups in both studies. Fig. $4 \mathrm{C}$ and $4 \mathrm{D}$ shows the immunoblotting for active caspase-3. The active caspase-3 expression in the HM or AA groups were increased compared to the VH group in both short-term $(2.8 \pm 0.6$ folds in the AA group and $3.3 \pm 0.4$ folds in the HM group, $p<0.05$ vs. VH group) and long-term studies (4.2 \pm 0.2 folds in the AA group and $1.8 \pm 0.1$ folds in the HM group, $p<0.05$ vs. VH group). Between HM and AA groups, there was no difference between two groups in short-term study, but HM group was lower than the AA group in long-term study.

\section{DISCUSSION}

In our study, we aimed to determine whether the presence of AA in a HM was a major factor in renal injury. To determine this, we performed an experimental study in mice to compare the nephrotoxic potential of HM containing AA and the AA compound administered alone. The results of our study clearly demonstrated that HM containing AA caused similar nephrotoxicity to that of $\mathrm{AA}$, and that AA concentration in HM was the major determinant of the severity of renal injury.

The purpose of short-term study was to test the acute toxic effects of HM. Based on AA dose in reported experimental model of acute AAN [6,8], we treated HM or AA $(5 \mathrm{mg} / \mathrm{kg})$ intraperitoneally in mice for 5 days. As expected, $\mathrm{HM}$ administration resulted in the typical features of acute kidney injury (AKI), including marked deterioration of renal function and acute tubular necrosis; moreover, the severity occurred at a similar level to that observed after administration of AA. In addition, the expression of NGAL, a biomarker of AKI, was increased in HM-treated mice and was comparable to the expression in AA-treated mice. This finding was consistent with previous reports that a high-dose AA caused AKI and that the major pathological finding was acute tubular necrosis [8-10].

We designed the long-term study to evaluate the chronic toxicity of HM based on the patient's prescription. We calculated the daily AA exposure from the prescribed dose and administered the same treatment duration as described in the patient's medical history. The long-term study revealed mild renal dysfunction, tubular atrophy and dilatation, and increased NGAL expression in both the HM and AA groups, with no significant difference between two groups. These findings suggested that long-term exposure to low-dose AA resulted in CKD. Collectively, AAN can present as AKI or CKD according to the concentration of AA or duration treatment of HM; this may be supported by a clinical report indicating that AAN results in a wide range of renal dysfunction based on the exposed dose and treatment 


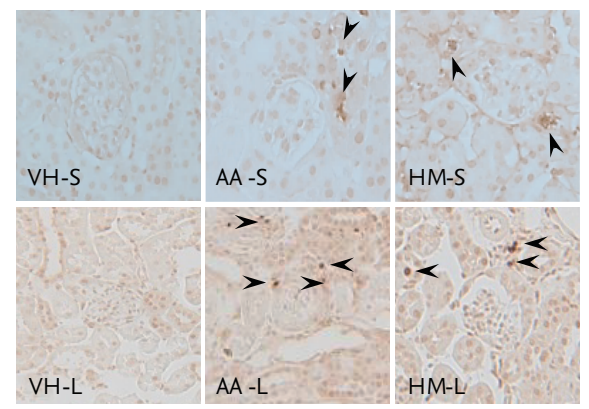

A

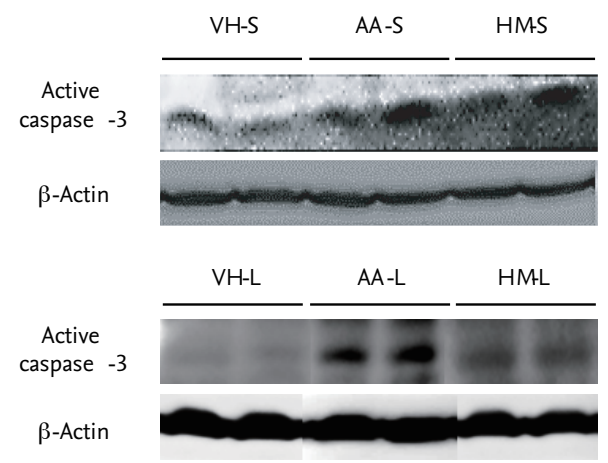

c

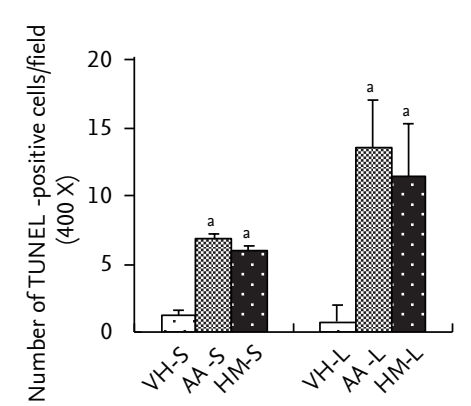

B

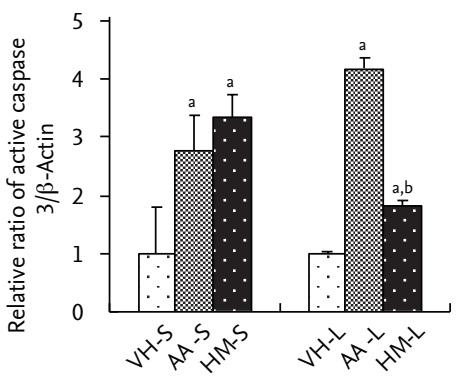

D
Figure 4. Comparison of apoptosis in aristolochic acid (AA) and herb medicine (HM) in the short-term and longterm studies. (A, B) Representative images and number of positively stained cells in the terminal deoxynucleotidyl transferase dUTP nick end labelling (TUNEL) assay (×400). (C, D) Representative images of immunoblotting and the quantification of active caspase-3 in the kidney; the expression is normalized to that of $\beta$-actin. The arrowheads indicate TUNEL-positive cells. The data are presented as the mean \pm $\mathrm{SE}$. VH, vehicle; $\mathrm{S}$, short-term treatment; L, long-term treatment. ${ }^{\mathrm{a}} \mathrm{A} p<$ 0.05 vs. the corresponding $\mathrm{VH}$ group, ${ }^{\mathrm{b}} \mathrm{A} p<0.05$ vs. the corresponding AA group. duration [4].

In long-term study, we could not develop renal fibrosis, common histologic finding of AAN [11], and it seems to be related to the treatment duration. We stopped experiment earlier (at day 30) than initial schedule (6 weeks) because of poor condition of experimental animals. Thus, we could observe only tubular injury (atrophy and dilatation). In this point, we expect that renal fibrosis can be developed with longer treatment. Another consideration in the long-term study is the discrepancy between pathology and renal function in the HM group. In spite of pathologic change (increased tubular atrophy and dilatation, and NGAL expression), renal function was normal (Scr, $0.22 \mathrm{mg} / \mathrm{dL}$ ). The reason for normal renal function in the HM group is unclear, but we speculate that antioxidant component in HM may reduce the nephrotoxic potential of AA [12].

The pathophysiological mechanisms through which AA induces renal injury are still largely unknown, but oxidative stress is reported as a common mechanism of AAN $[13,14]$. Therefore, we compared the oxidative stress and the resulting cell death in HM- and AA-treated mice. In this study, we found that HM exerted a similar degree of oxidative stress (8-OHdG and MnSOD) and apoptotic cell death (TUNEL-positive cells and activated caspase-3) compared with AA; similarly, mitochondrial damage induced by HM was also similar to that induced by the administration of AA alone in both the short-term and long-term studies. These findings confirmed that, in terms of oxidative stress, HM induces a similar degree of injury to AA, suggesting that oxidative stress induced by HM resulted in mitochondrial damage, apoptosis, and caspase-3 activation, which may lead to progressive tubular atrophy and interstitial fibrosis [11,14].

The results in our study illustrate the comparable nephrotoxicity of $\mathrm{HM}$ and $\mathrm{AA}$; however, there are some limitations. First, there might be a selection bias as the HM obtained from a single source. Second, we did not 
consider species-specific toxicity of AA [15]. Therefore, the results of our study in mice should be interpreted with caution in humans. Third, the AA dose $(0.073 \mathrm{mg} /$ $\mathrm{kg}$, twice a day) for long-term study was lower than dose ( 4 or $5 \mathrm{mg} / \mathrm{kg}$ ) for experimental AAN. But, our study suggests that long-term exposure to low dose AA can develop AAN.

In conclusion, HM containing AA had a similar nephrotoxic potential to that of AA.

\section{KEY MESSAGE}

1. The nephrotoxic potential of herbal medicine containing aristolochic acid is similar to that of aristolochic acid itself.

2. The concentration of aristolochic acid in herbal medicine is the major determinant of the severity of renal injury.

3. Long-term exposure to low dose aristolochic acid can develop aristolochic acid nephropathy.

\section{Conflict of interest}

No potential conflict of interest relevant to this article was reported.

\section{Acknowledgments}

This study was supported by a grant of the Korean Health Technology R\&D Project, Ministry for Health and Welfare, Republic of Korea (HI14C3417).

\section{REFERENCES}

1. Vanherweghem JL, Depierreux M, Tielemans C, et al. Rapidly progressive interstitial renal fibrosis in young women: association with slimming regimen including Chinese herbs. Lancet 1993;341:387-391.

2. Lord GM, Tagore R, Cook T, Gower P, Pusey CD. Nephropathy caused by Chinese herbs in the UK. Lancet 1999;354:481-482.

3. Lee $\mathrm{S}$, Lee T, Lee B, et al. Fanconi's syndrome and subsequent progressive renal failure caused by a Chinese herb containing aristolochic acid. Nephrology (Carlton)
2004;9:126-129.

4. Yang L, Su T, Li XM, et al. Aristolochic acid nephropathy: variation in presentation and prognosis. Nephrol Dial Transplant 2012;27:292-298.

5. Tian Y, Yang Y, Gao L, et al. Expression of histone deacetylase-1 and p3oo in aristolochic acid nephropathy models. Toxicol Mech Methods 2014;24:377-384.

6. Savransky V, Molls RR, Burne-Taney M, Chien CC, Racusen $\mathrm{L}$, Rabb $\mathrm{H}$. Role of the T-cell receptor in kidney ischemia-reperfusion injury. Kidney Int 2006;69:233-238.

7. Lim SW, Doh KC, Jin L, et al. Oral administration of ginseng ameliorates cyclosporine-induced pancreatic injury in an experimental mouse model. PLoS One 2013;8:e72685.

8. Wu J, Liu X, Fan J, et al. Bardoxolone methyl (BARD) ameliorates aristolochic acid (AA)-induced acute kidney injury through Nrf2 pathway. Toxicology 2014;318:22-31.

9. Decleves AE, Jadot I, Colombaro V, et al. Protective effect of nitric oxide in aristolochic acid-induced toxic acute kidney injury: an old friend with new assets. Exp Physiol 2016;101:193-206.

10. Dai XY, Zhou L, Huang XR, Fu P, Lan HY. Smad7 protects against chronic aristolochic acid nephropathy in mice. Oncotarget 2015;6:11930-11944.

11. Zhao YY, Wang HL, Cheng XL, et al. Metabolomics analysis reveals the association between lipid abnormalities and oxidative stress, inflammation, fibrosis, and Nrf2 dysfunction in aristolochic acid-induced nephropathy. Sci Rep 2015;5:12936.

12. Ban TH, Min JW, Seo C, et al. Update of aristolochic acid nephropathy in Korea. Korean J Intern Med 2018;33:961969.

13. Matsui K, Kamijo-Ikemorif A, Sugaya T, Yasuda T, Kimura K. Renal liver-type fatty acid binding protein (L-FABP) attenuates acute kidney injury in aristolochic acid nephrotoxicity. Am J Pathol 2011;178:1021-1032.

14. Zeng Y, Li S, Wu J, et al. Autophagy inhibitors promoted aristolochic acid I induced renal tubular epithelial cell apoptosis via mitochondrial pathway but alleviated nonapoptotic cell death in mouse acute aritolochic acid nephropathy model. Apoptosis 2014;19:1215-1224.

15. Huljic S, Bruske EI, Pfitzenmaier N, O'Brien E, Dietrich DR. Species-specific toxicity of aristolochic acid (AA) in vitro. Toxicol In Vitro 2008;22:1213-1221. 